\title{
Micromechanical approaches to understand dwell fatigue: from titanium a-b microstructures to disc thermal alleviation
}

\author{
Zebang Zheng, Zhen Zhang, Ben Britton, Fionn Dunne \\ Imperial College, London, UK
}

\begin{abstract}
$\underline{\text { Abstract }}$
Micro-pillar tests on $\alpha$ and $\alpha-\beta$ colony Ti alloys in combination with crystal plasticity finite element analysis has enabled the extraction of $a$ and $b$ phase slip strength and rate sensitivity properties. Faithfully representative $\alpha-\beta$ microstructure polycrystal plasticity models have then been established in order to investigate dwell fatigue in isothermal rig test behaviour and anisothermal thermomechanical flight loading conditions. The role of thermal alleviation in diminishing dwell sensitivity has been demonstrated.
\end{abstract}

\section{Introduction}

Cold dwell fatigue was first recognised through an air accident in the 1970s due to the failure of two titanium fan disks in Rolls-Royce RB211 engines [1,2]. A significant lifetime reduction, known as dwell debit, occurs when titanium alloys are subjected to cyclic loading with stress hold at maximum magnitude (dwell period) in each cycle at low temperatures [3-6]. Dwell sensitivity of titanium alloys has been an important research interest since then.

Facet crack nucleation on basal slip planes of hexagonal closed packed (HCP) crystals is argued to be responsible for the early service failure of near $\alpha$ titanium alloys [7]. The local crystallographic orientation combination with respect to the loading direction was found to cause load shedding during the dwell period in which stresses are redistributed from 'soft' grains into the adjacent 'hard' grains [5]. This local creep behaviour results in high grain boundary stresses [8] and may induce the subsequent crack nucleation. Much work has been carried out to understand the mechanistic basis of the load shedding phenomena. Hasija et al. [5] first reported the stress redistribution from soft to hard-orientated grains in single $\alpha$ phase Ti-6Al using crystal plasticity (CP) modelling. Huang et al. [9] demonstrated the load partitioning between the soft (matrix) and hard (precipitates) grains due to the creep behaviour using in-situ neutron diffraction technique. Sinha et al. [7] studied near a Ti-6242 alloy and have shown the prismatic slip accumulated in the soft grain results in dislocation pileups and hence inducing elevated stress fields more likely to initiate cracks on basal planes in the adjacent hard grain. Modelling work by Dunne et al. $[10,11,12]$ and Ghosh et al. $[13,14,15]$ has shown the local stress development under dwell fatigue loading at room temperature using homogenised $\alpha$ phase CP models. Joseph et al. [16] and Zheng et al. [17] investigated the effect of slip transfer through interfaces on fatigue behaviour of titanium 
alloys. Bache et al. [18] found that the dwell debit is reduced by applying lower peak hold stress. Ozturk et al. [19] using CP modelling also demonstrated that the number of cycles to nucleate facets increases with decreasing the dwell stress.

The modelling work by Zhang et al. [20] found that load shedding in $\alpha$ Ti-6Al alloy increases with temperature from $20^{\circ} \mathrm{C}$ and peaks at about $120^{\circ} \mathrm{C}$ followed by a diminution to zero at about $220^{\circ} \mathrm{C}$. This results from the influence of temperature on both the slip system strengths (CRSSs) and strain rate sensitivity. Ozturk et al. [21] using homogenised $\alpha$-HCP 3D crystal plasticity finite element modelling with the grain size distribution and orientation distribution fully recognised to investigate the fatigue crack nucleation under isothermal conditions between $300^{\circ} \mathrm{C}$ and $600^{\circ} \mathrm{C}$. The simulation results are also able to reproduce the diminution of dwell sensitivity at elevated temperatures as observed experimentally. The atomistic simulations carried out by Ready et al. (Ready et al., 2017) demonstrated that Mo atoms in titanium alloys are not able to trap the vacancies for long enough at room temperatures in order to give rise to the observed strain rate sensitivity. This is important in demonstrating that it is not the chemistry (or more particularly the molybdenum) which gives rise intrinsically to the differing dwell behaviours of alloys Ti-6242 and Ti-6246. Zheng et al. [22] using discrete dislocation plasticity (DDP) investigated the temperature sensitivity of dwell fatigue in Ti-6242 and Ti-6246 alloys assuming homogenised $\alpha-\mathrm{HCP}$ polycrystal representations in both cases. Similar behaviour of temperaturedependent load shedding as for Ti-6Al alloy was found for Ti-6242, and alloy Ti-6246 was found to show dwell sensitivity at higher temperatures of $300^{\circ} \mathrm{C}$. When the $\alpha-\beta$ basketweave microstructure is fully accounted for in the latter material, it is anticipated that the alloy would not be expected to show any dwell sensitivity.

This paper addresses dwell fatigue in isothermal rig test conditions as well as for thermomechanical loading representative of in-service flight conditions using faithfully representative $\alpha-\beta$ Ti microstructures and assesses the role of temperature in thermal alleviation.

\section{Micro-pillar experiments and crystal plasticity modelling}

Material (Ti-6242) was supplied by IMR (Institute of Metal Research, China). Micro-pillars with square section were fabricated using FIB (focused ion beam) in both pure $\alpha$ and $\alpha-\beta$ microstructures with a width of $2 \mu \mathrm{m}$ and height of $5 \mu \mathrm{m}$ as shown schematically in Figure 1 , together with an example $\alpha-\beta$ pillar. Stress relaxation through compressive displacement hold tests was investigated on micro $\alpha$ and $\alpha-\beta$ pillars in order to determine phase properties. The pillars were tested within in-situ SEM using an Alemnis nano-indentation platform, enabling direct observation of crystal slip during deformation where slip bands on the surface are generated. The compression was imposed by an indenter with a flat punch (10 $\mu \mathrm{m}$ diameter, set on top of a $60^{\circ}$ cone) [23]. 


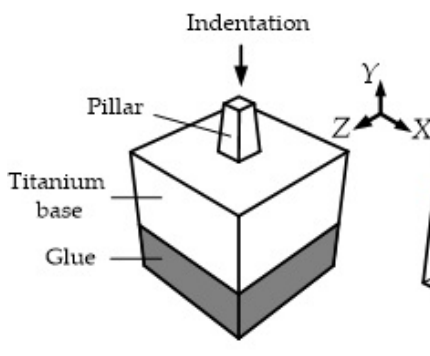

(a)

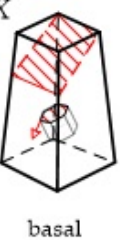

(b)

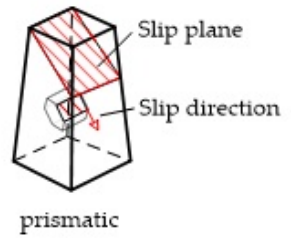

(c)

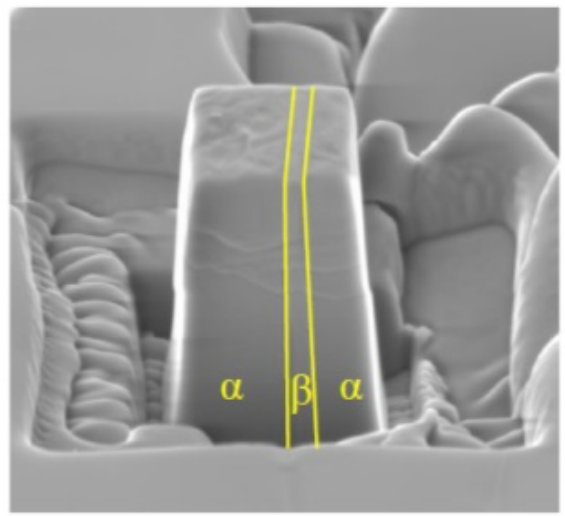

Figure 1 (a) Micro-pillar schematic with crystal orientation for expected (b) basal and (c) prismatic slip in pure $\alpha$ phase pillars, (d) $\alpha-\beta$ pillar.

The micro-pillars were explicitly modelled utilising crystal plasticity finite element methods in order to extract out the key properties including slip strengths, and slip strain rate sensitivities, governed by an activation energy for thermally-activated dislocation escape from obstacles and an associated activation volume. The details of the crystal plasticity modelling and property extraction may be found in [24,25]. An example of the results obtained for the pillar shown in Figure 1 is given in Figure 2 which shows the experimentally observed slip together with the calculated crystal plasticity pillar displacement field.
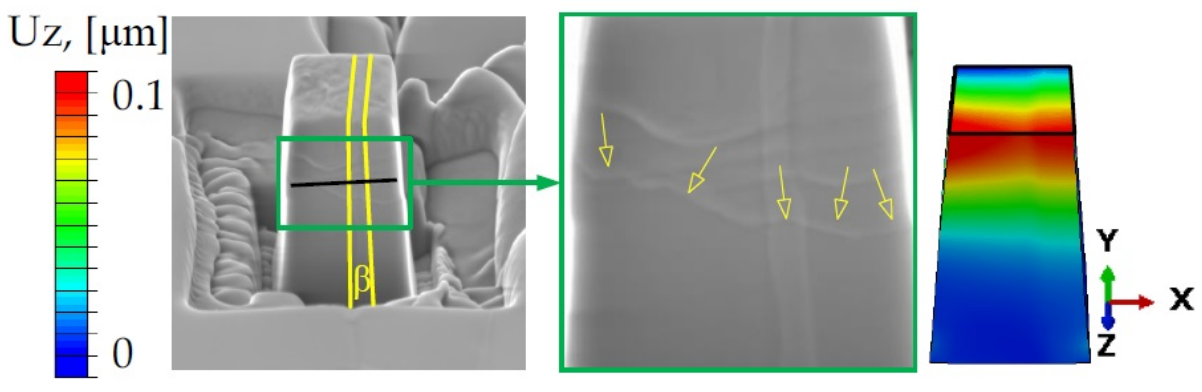

Figure 2 Experimental and crystal plasticity calculated behaviour for an $\alpha-\beta$ micro-pillar. Crystal calculations show z-displacement fields. The $\beta$-phase regions are highlighted in yellow, and the additional black line indicates predominant slip planes. The slip lines are highlighted by yellow arrows in the zoom-in window.

The full property evaluation for the $\alpha$ and $\beta$ phases at $20^{\circ} \mathrm{C}$ may be found in $[24,25]$ and a key outcome is the very significant strain rate sensitivity of both phases at $20^{\circ} \mathrm{C}$ which, under conditions of strain hold 
periods during loading, leads to very substantive stress relaxations. Conversely, under stress hold periods, the alloys are found to demonstrate very significant creep which depends strongly on crystal orientation with respect to loading. Crystal c-axes parallel to load inhibit slip and shows little creep. Orientations facilitating basal or prismatic slip give rise to considerable creep under load with the former showing the higher strain rate sensitivity. These rate-sensitive behaviours are argued to be key to understanding the cold dwell fatigue phenomenon together with its thermal alleviation, which are addressed next.

\section{Dwell fatigue and thermal alleviation}

We firstly address local multi-axial loading conditions corresponding to experimental (disc spin) tests shown in Figure 3 at $80^{\circ} \mathrm{C}$ with hoop, axial and radial stresses shown which result from full scale experimental spin testing. That is, at every location of interest within the disc shown in Figure 3(a) and (b), the local multiaxial stresses during a loading cycle are extracted from a full component-level elastic finite element analysis. These extracted local stress histories are then applied to representative polycrystal plasticity RVE as boundary conditions, as indicated in Figure 3. It is therefore assumed that the loading cycle boundary conditions remain unchanged during subsequent cycling. The three rig tests shown in Figure 3(c) and (d) all lead to failure in the experimental observations resulting from dwell facet nucleation at hard/soft grain orientation combinations at the locationsshown in Figure 3.
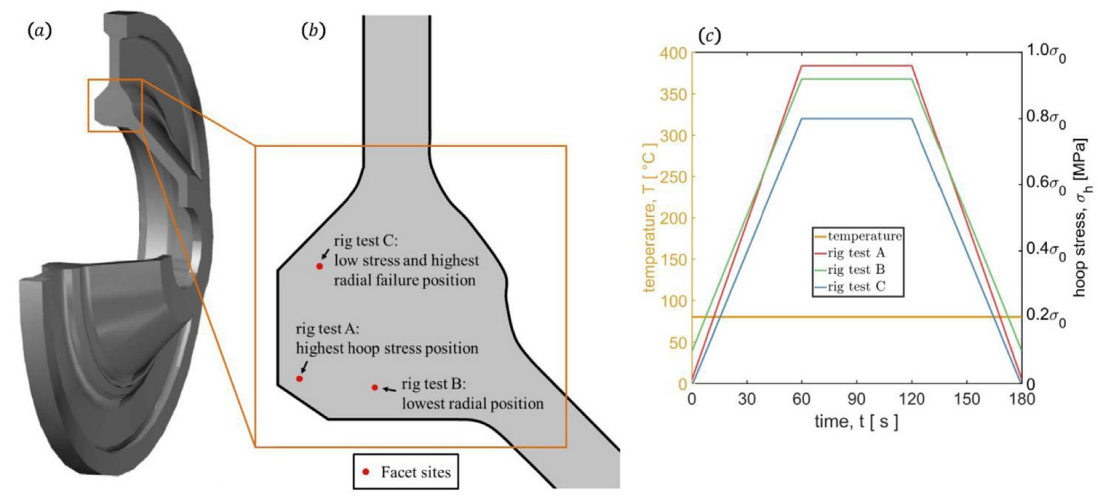

Figure 3 (a) Schematic illustration of a compressor disc in rig spin tests with 1/4 cutaway [3]. (b) The observed facet locations for three rig tests, A, B and C. (c) Loading histories (only the primary hoop stress is shown; axial and radial stresses also develop and are included in the analysis) of the three rig tests.

A polycrystal plasticity finite element model has been established which approximately replicates a region of the two phase morphology of the IMI834 bimodal and lamellar $\alpha-\beta$ structure as shown in Figure 4. The model incorporates $39 \alpha$ grains with different crystal orientations and a hard grain crystallographic orientation is assigned to the primary $\alpha$ grain located about in the centre of the region modelled, and a neighbouring secondary $\alpha$ grain is assigned a soft crystallographic orientation favouring a-prismatic slip with respect to the hoop stress loading direction. Crystal orientations of the remaining $\alpha$ 
grains are chosen to achieve an overall random texture as shown in Figure 3(d) to reflect the texture associated with the disc test samples. The Burgers Orientation Relationship (BOR) between $\alpha$ and $\beta$ phase orientations is faithfully satisfied for all neighbouring $\alpha-\beta$ laths in the model representation. The microstructure is extruded along the thickness direction in the dual phase model as shown in Figure 4(e). The model contains 178,422 ten-noded tetrahedral elements (C3D10). It is noted that four-noded tetrahedral elements can sometimes exhibit an overly stiff response resulting from volumetric locking and in the present dual phase model, ten-noded tetrahedral elements are used to avoid the locking problem.

Here we use this dual phase model to study the three isothermal rig spin tests and in addition, two thermo-mechanical loadings representative of engine in-service conditions and discuss the mechanisms of thermal alleviation on cold dwell fatigue resulting from the anisothermal temperature loading.
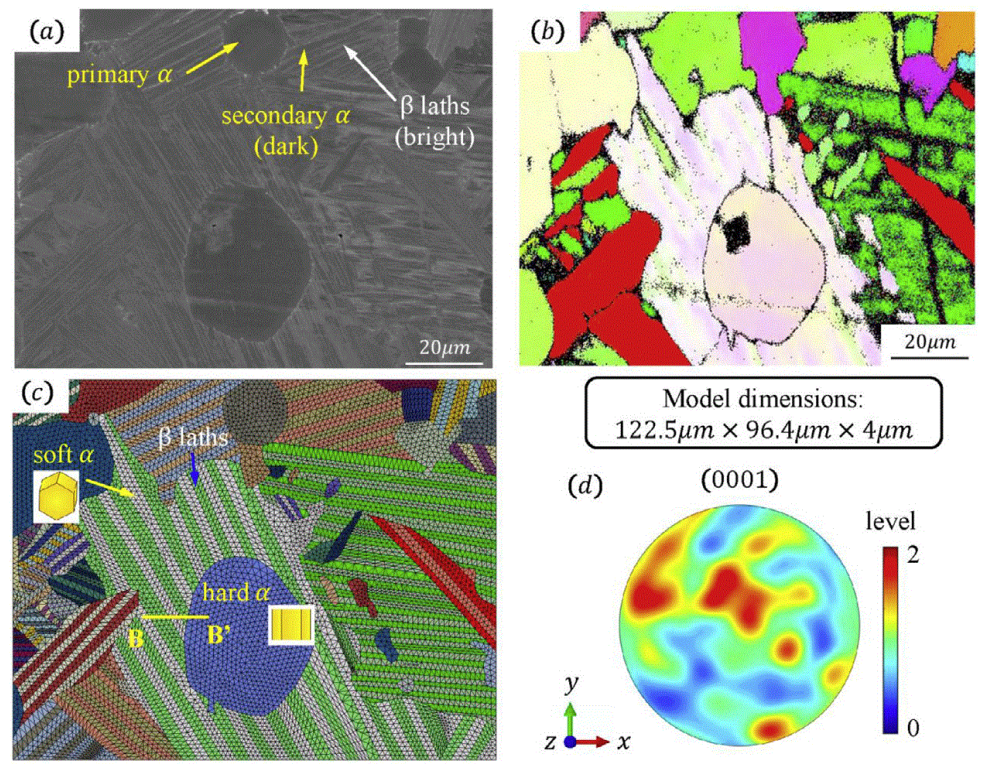

(e)

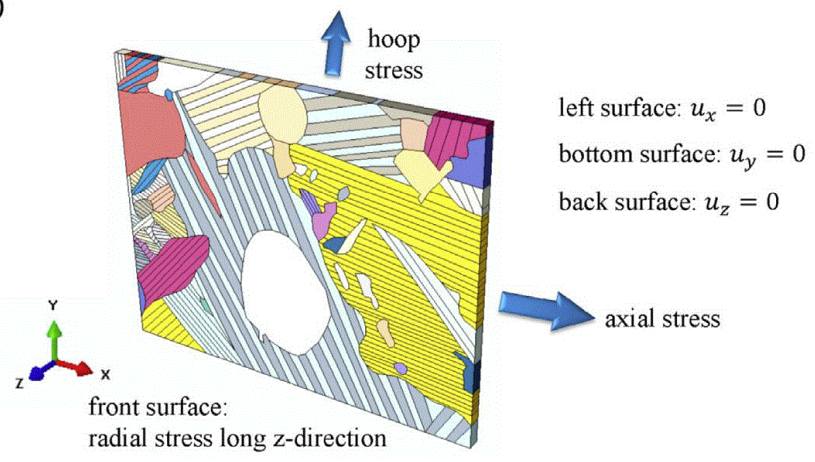


Figure 4 (a) SEM image of $\alpha-\beta$ alloy [26] shows the bimodal microstructure; (b) the corresponding EBSD map; (c) front surface of the dual phase polycrystal CP model with $\alpha-\beta$ morphology explicitly represented (d) pole figure showing the overall random texture assigned in the $\alpha$ grains in the model, and (e) the imposed boundary conditions.

The three rig spin test loading conditions shown in Figure 3 are applied to the dual phase model shown in Figure 4(e) with the hoop stress along the y-direction and axial and radial stresses along the $\mathrm{x}$ - and $\mathrm{z}$ directions respectively. The applied stresses are lowest for rig test $\mathrm{C}$, but even for this case, the results demonstrate that the explicit inclusion of the complex $\alpha-\beta$ lamellar microstructure has led to stress states which are sufficiently high to drive the onset of local slip. This makes clear the point that in order to capture slip-driven damage processes occurring during dwell fatigue at realistic loading levels (i.e. inservice aero-engine conditions), it is essential to include microstructural detail. The higher loading levels for rig spin tests $\mathrm{A}$ and $\mathrm{B}$ naturally give rise to greater slip activation. The stress and effective plastic strain distributions along path B-B' (Figure 4(c)) are examined before and after the stress hold periods in order to give indicative load shedding behaviour, and the results are summarised in Figure 5 for rig test A. Note that path B-B' is chosen arbitrarily and does not identify those locations giving rise to the highest stresses resulting from load shedding (see later). The peak stresses developed at the soft-hard grain boundaries for all three tests are higher after the dwell period, thus showing the activation of load shedding in this material at stresses relevant to in-service conditions. The results also show the role of the $\beta$ phase laths in perturbing the stress distributions in the soft grains, leading to bands of lower stress (within the $\beta$ laths) such that higher stresses are generated elsewhere in the soft $\alpha$ laths and hard $\alpha$ grains in order to maintain conditions of equilibrium during the stress hold period.
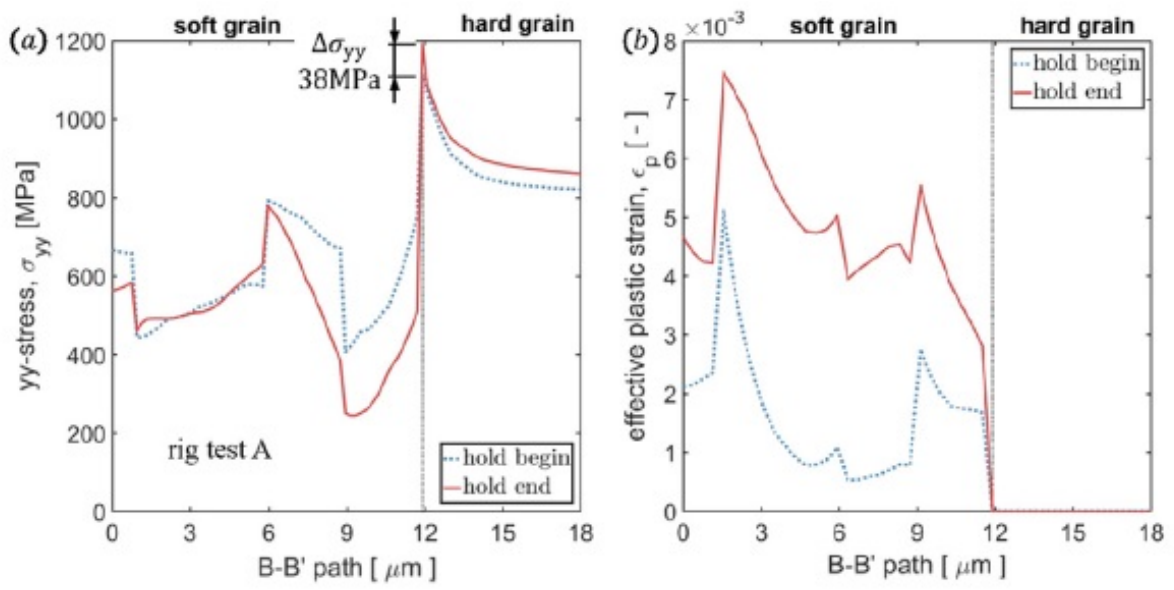

Figure 5 Stress and effective plastic strain distributions along path B-B' (Figure 4(c)) for rig test A before and after the stress hold (dwell) shown in Figure 3 [26]. 
In order to investigate further the interactions of temperature and stress in cold dwell fatigue, and in particular with respect to the isothermal rig tests and (anisothermal) in-service engine conditions, a peak (hard grain basal) stress against operating temperature map has been generated as shown in Figure 6, in which three regimes identified as dangerous, threatening and safe have been introduced with respect to dwell facet nucleation. Superimposed on the map are the thermo-mechanical loadings associated with the first cycle only of each of four loading scenarios considered: the three rig tests (A, B and C) and the inservice (labelled Redline) conditions. The criteria defining the regions are that a basal (hard grain) stress greater than $1200 \mathrm{MPa}$ is taken to be a necessary condition for facet nucleation (determined from rig spin tests [3]), that a temperature in excess of $200^{\circ} \mathrm{C}$ inhibits facet nucleation [20,22], and that soft grain slip is also a prerequisite for facet nucleation (which defines the elastic region shown). However, the diagram is not yet complete in that it shows the loading histories with respect to the demarcations but only for a single first loading cycle. Earlier, it was made clear that there are conditions giving rise to progressive cyclically increasing hard grain basal stress such that a given number of cycles is required in order to drive the basal stress higher than the critical 1200MPa. Hence the region identified as threatening (yellow) has been included. In addition, for those loading cases for which a progressively increasing hard grain basal stress occurs (rig tests A, B and C), an upward arrow has been included at the loading history termination; where the hard grain basal stress is unchanged (i.e. stable) with further cycles, a cross is shown (in-service Redline).

Hence a peak stress in the loading history entering the red zone indicates a propensity to nucleate a dwell facet. The yellow zone represents the strain rate sensitive temperatures, and if the basal stress developed is located in this region, even if its current magnitude is lower than the critical stress, it should be treated as a threat because the basal stresses are ratcheting up (due to cold creep in the adjacent soft grain during dwell) to exceed the critical stress of $1200 \mathrm{MPa}$ with further loading cycles. The material behaviour relevant to the yellow zone has been studied in some detail [22] in which thermally activated dislocation escape from obstacles during stress dwell is shown via discrete dislocation analyses to lead to dislocation pile-up at the hard-soft grain boundaries, leading to a gradual cycle by cycle increase in the hard grain basal stress, supporting the $\mathrm{CP}$ studies reported here. The green zone in Figure 8 indicates the safe zone for which the loading is not anticipated to give rise to dwell fatigue. In this zone, the basal stress is not high enough to nucleate a dwell facet in the first cycle, and the stress cannot increase in subsequent cycles due to the diminution of cold creep accumulation and load shedding during dwell at temperature in excess of $\sim 200^{\circ} \mathrm{C}$. The mechanistic basis for this was also studied using discrete dislocation analyses [22] showing that in these circumstances, dislocation escape from obstacles occurs very rapidly (as a result of a low effective energy barrier to dislocation escape) such that equilibrium is achieved before stress dwell inhibiting load shedding. Titanium alloys subjected to these kinds of loading conditions do not display dwell sensitivity. 


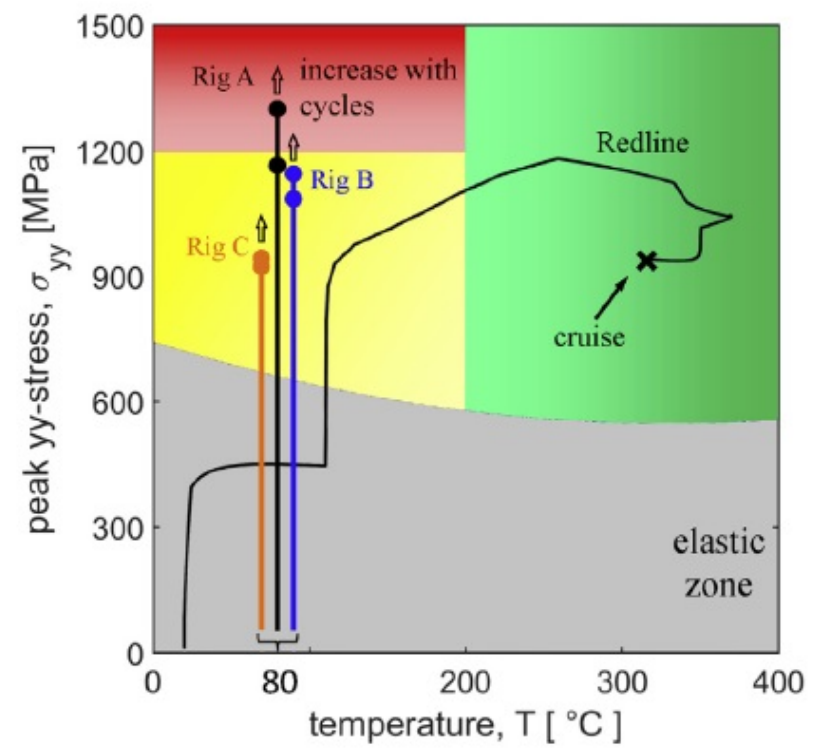

Figure 6 Peak basal stress against operating temperature map demarcated into dangerous (red), potential threat (yellow) and safe (green) regions with respect to dwell facet nucleation. The CP determined results for the (isothermal) rig spin tests and in-service Redline test after one loading cycle are superimposed.

\section{Conclusions}

Dwell fatigue in isothermal rig spin tests and thermomechanical loading representative of in-service flight conditions has been assessed and the role of thermal alleviation in diminishing dwell sensitivity for in-service elevated temperature conditions demonstrated.

\section{$\underline{\text { References }}$}

[1] Bache, MR. Int. Jnl. Fatigue. 25 (2003) 1079-1087.

[2] Song, Z, Hoeppner, DW. Intl. Jnl. Fatigue. 11 (1989) 85-90.

[3] Cuddihy, MA, Stapleton, A, Williams, S, Dunne, FPE. Intl. Jnl. of Fatigue 97 (2017) 177-189.

[4] Evans, WJ, Bache, MR. Intl. Jnl. Fatigue 16 (1994) 443-452.

[5] Hasija, V, Ghosh, S, Mills, MJ, Joseph, DS. Acta Mat. 51 (2003) 4533-4549.

[6] Qiu, J, Ma, Y, Lei, J., Liu, Y, Huang, A, Rugg, D, Yang, R. Metallurgical and Materials Transactions A. 45 (2014) 6075-6087. 
[7] Sinha, V, Mills, MJ, Williams, JC, Spowart, JE. Metallurgical and Materials Transactions A. 37 (2006)1507-1518.

[8] Zheng, Z, Balint, DS, Dunne, FPE. Intl. Jnl. Plasticity 87 (2016) 15-31.

[9] Huang, S, Brown, DW, Clausen, B, Teng, Z, Gao, Y, Liaw, PK. Met. Mats. Trans. 43 (2012) 14971508 .

[10] Dunne, FPE, Rugg, D. Length scale, orientation and morphology effects in fatigue crack nucleation in polycrystals. Advances in Heterogeneous Material Mechanics 2008. June 3-8, 2008, Huangshan, China. DEStech Publications, Inc, p. 145.

[11] Dunne, FPE, Rugg, D. Fat, Fract. Eng. Mats \& Struct. 31 (2008) 949-958.

[12] Dunne, FP.E, Walker, A, Rugg, D. Proc. R. Soc. 463 (2007) 1467-1489.

[13] Ghosh, S, Anahid, M. Intl. Jnl. Plast. 47 (2013) 182-201.

[14] Ghosh, S, Chakraborty, P. Intl. Jnl. Fatigue 48 (2013) 231-246.

[15] Venkataramani, G, Kirane, K, Ghosh, S. Intl. Jnl. Plasticity 24 (2008) 428-454.

[16] Joseph, S, Bantounas, I, Lindley, TC, Dye, D. Intl. Jnl. Plasticity 100 (2018) 90-103.

[17] Zheng, Z, Waheed, S, Balint, DS, Dunne, FPE. Intl. Jnl. Plasticity 104 (2018) 23-38.

[18] Bache, MR, Cope, M, Davies, HM, Evans, WJ, Harrison, G. Intl. Jnl. Fatigue 19 (1997) 83-88.

[19] Ozturk, D, Pilchak, AL, Ghosh, S. Scripta Mat. 127 (2017) 15-18.

[20] Zhang, Z, Cuddihy, MA, Dunne, FPE. Proc. R. Soc. Lond. (2015) 471.

[21] Ozturk, D, Shahba, A, Ghosh, S. Fat. Fract. Eng. Mats. Struct. 39 (2016) 752-769.

[22] Zheng, Z, Balint, DS, Dunne, FPE. Jnl. Mech. Phys. Solids. 107 (2017) 185-203.

[23] Jun, TS, Sernicola, G, Dunne, FPE, Britton, T. Mats. Sci. Eng. A. 649 (2016) 39-47.

[24] Zhang, Z, Jun, T, Britton, TB, Dunne, FPE. Acta Mat. 118 (2016) 317-330.

[25] Zhang, Z, Jun, TS, Britton, TB, Dunne, FPE. Jnl. Mech. Phys. Solids. 95 (2016) 393-410.

[26] Zheng, Z, Stapleton, A, Fox, K, Dunne, FPE. Intl. Jnl. Plasticity. 111 (2018) 234-252. 
[27] Anahid, M, Chakraborty, P, Joseph, D, Ghosh, S, Mod. Sim. Mats. Sci. Eng.17, (2009).

[28] Anahid, M, Samal, MK, Ghosh, S. Jnl. Mech. Phys. Solids. 59 (2011) 2157-2176. 